\title{
Toll-like receptor 4 and its associated proteins as prognostic factors for HCC treated by post-radiotherapy surgery
}

\author{
ZHI-FENG WU ${ }^{1 *}$, YING WANG $^{2 *}$, PING YANG $^{1 *}$, JIA-ZHOU HOU $^{1}$, \\ JIAN-YING ZHANG ${ }^{1}$, YONG HU ${ }^{1}$ and ZHAO-CHONG ZENG ${ }^{1}$ \\ ${ }^{1}$ Department of Radiation Oncology, Zhongshan Hospital, Fudan University; ${ }^{2}$ Department of \\ Ultrasonography, Huashan Hospital, Fudan University, Shanghai 200032, P.R. China
}

Received December 13, 2015; Accepted October 18, 2017

DOI: $10.3892 / \mathrm{ol} .2018 .8583$

\begin{abstract}
Locally advanced hepatocellular carcinoma (HCC) treated by radiotherapy (RT) may be suited for further treatment with surgery. As a critical mediator of the post-RT immune response, Toll-like receptor 4 (TLR4) and its associated proteins may serve as prognostic factors for patients with HCC treated by post-RT surgery. In the present study, a total of 20 patients with HCC treated by post-RT surgery were enrolled. Resected tumor and peritumoral liver tissues were used to construct tissue microarrays that were assessed with immunohistochemical staining for the expression levels of TLR4, tumor necrosis factor-related apoptosis-inducing ligand (TRAIL) and vascular endothelial growth factor receptor 2 (VEGFR2). The overall (OS) and disease-free (DFS) survival outcomes for each patient were assessed, and the severity of radiation-induced liver diseases (RILDs) was detected. The patients with low TLR4 or TRAIL expression exhibited significantly better OS times than those with high TLR4 ( $\mathrm{P}=0.003)$ or TRAIL $(\mathrm{P}=0.007)$ expression, whereas the median DFS times for patients with low VEGFR2 or TRAIL were significantly longer than those with high VEGFR2 $(\mathrm{P}=0.003)$ or TRAIL $(\mathrm{P}=0.008)$ expression. No significant differences in OS or DFS times were identified according to the expression of TLR4, VEGFR2 or TRAIL in peritumoral liver
\end{abstract}

Correspondence to: Professor Zhao-Chong Zeng, Department of Radiation Oncology, Zhongshan Hospital, Fudan University, 180 Feng Lin Road, Shanghai 200032, P.R. China

E-mail: zeng.zhaochong@zs-hospital.sh.cn

*Contributed equally

Abbreviations: HCC, hepatocellular carcinoma; TLR4, Toll-like receptor 4; RT, radiotherapy; TMA, tissue microarray; OS, overall survival; DFS, disease-free survival; RILD, radiation-induced liver disease; TRAIL, tumor necrosis factor-related apoptosis-inducing ligand; VEGFR2, vascular endothelial growth factor receptor 2

Key words: hepatocellular carcinoma, post-radiotherapy surgery, Toll-like receptor 4, vascular endothelial growth factor receptor 2, tumor necrosis factor-related apoptosis-inducing ligand tissue, although more severe RILDs were identified in patients with the high expression of these factors in the peritumoral liver tissue post-RT $(\mathrm{P}<0.05)$. Therefore, the expression levels of TLR4 and its associated proteins in HCC tumors may be suitable as prognostic factors for patients with HCC treated by post-RT surgery. The inhibition of TLR4, VEGFR2 and TRAIL expression in HCC and non-tumor liver tissue may lessen the severity of RILDs and improve survival outcomes in the future.

\section{Introduction}

Hepatocellular carcinoma (HCC) is one of the most malignant types of cancer and is associated with a poor prognosis. Although surgical resection is potentially curative, the postoperative recurrence rate is $>50 \%(1,2)$. Due to receiving a HCC diagnosis at an advanced stage, a number of patients with HCC are not suited for surgical treatment. At present, radiotherapy (RT) is recommended as the treatment for patients with locally advanced HCC $(3,4)$, and certain patients can then be treated by surgical resection, post-RT. An important aspect of HCC therapy is identifying which patients will benefit from post-RT surgical resection.

Toll-like receptor 4 (TLR4) is an initiator of the innate immune response that recognizes molecules derived from pathogens (PAMPs) and endogenous danger signals (DAMPs) (5). The excessive activation of TLR4 signaling, as may be stimulated by DAMPs derived from distress or injury in tissues following RT, may induce liver damage (6), and further influence the apoptosis, proliferation, invasion and metastasis of HCC $(7,8)$. Our previous studies identified that the TLR4-dependant immune response may promote radiation-induced liver diseases (RILDs) via enhancing the expression of certain proteins in mice, including tumor necrosis factor-related apoptosis-inducing ligand (TRAIL) and vascular endothelial growth factor receptor 2 (VEGFR2) $(9,10)$. However, the clinical value of these potentially influencing factors in estimating the prognosis for patients with HCC treated by surgical resection post-RT remains unclear.

The purpose of the present study was to evaluate the effect of TLR4-associated proteins on the response of HCC to RT followed by surgery. Therefore, the individual characteristics and prognoses of patients with HCC treated by surgical 
resection post-RT were considered, and the expression levels of TLR4, TRAIL and VEGFR2 in HCC and peritumoral liver tissue samples from the patients were assessed using immunohistochemistry (IHC) assays on liver resection specimens.

\section{Materials and methods}

Patients. Between July 2005 and October 2013,20 patients with HCC who underwent surgical resection post-RT at Zhongshan Hospital (Shanghai, China) were selected for this study. All the recruited patients exhibited stage III disease, including 19 males and 1 female, with ages ranging from 36-69 and a mean age of 54 years. Tumor staging was performed according to the 2002 International Union Against Cancer system (11). The study was approved by the Zhongshan Hospital Research Ethics Committee, and all patients provided written consent for their inclusion in research.

The selection criteria for the patients recruited into the study were as follows: i) Patients were diagnosed with stage III HCC prior to RT, confirmed by histology or a serum $\alpha$-fetoprotein level $\geq 400 \mathrm{ng} / \mathrm{ml}$, with a typical clinical presentation; ii) surgery had been judged to be unsuitable as a primary treatment; iii) intrahepatic tumors were treated with RT; iv) as assessed by the Response Evaluation Criteria In Solid Tumors (12), the tumor response to RT had been judged as a partial response (PR) prior to surgery; and v) intrahepatic tumors underwent surgical resection following RT. In addition, as transarterial chemoembolization (TACE) is typically used for the palliation of unresectable HCC and numerous patients had been treated with TACE, patients that had been previously treated with TACE were not excluded from this retrospective research.

Therapeutic strategy. Intrahepatic tumors were treated with external beam RT (EBRT) using a linear accelerator or helical tomotherapy. The plans for RT were produced using a treatment planning system (TPS) computer. The tumor dose was 46-53.5 Gy in 10-25 fractions. To assess the tumor response to RT, abdominal enhanced computed tomography (CT) or magnetic resonance imaging (MRI) was performed pre-and post-RT for each patient. Prior to the surgery, patients included in this study were all judged to be operable by their surgeons. Hepatectomy was then applied for the further treatment of intrahepatic tumors subsequent to RT. In addition, patients could be treated with or without TACE pre- or post-surgery. TACE could also serve as abridging therapy prior to RT in some patients with HCC, especially for those with large tumors, because the minimum normal liver volume during RT (minus the gross tumor volume) should be more than $700 \mathrm{ml}$ to avoid RILD. Following treatment with TACE, significant tumor regression may occur, and some patients could be further treated with RT. Furthermore, the complete tumor response rate of TACE is unsatisfactory $(13,14)$, meaning that HCC may not be cured by TACE alone. In order to improve efficacy, some HCC patients may switch to RT following TACE treatment. Therefore, each patient's treatment history, with or without TACE, would be analyzed in the present study.

For example (Fig. 1), a patient with HCC presented with a large mass, which was unsuited for primary treatment by surgical resection (Fig. 1A), and was therefore treated with

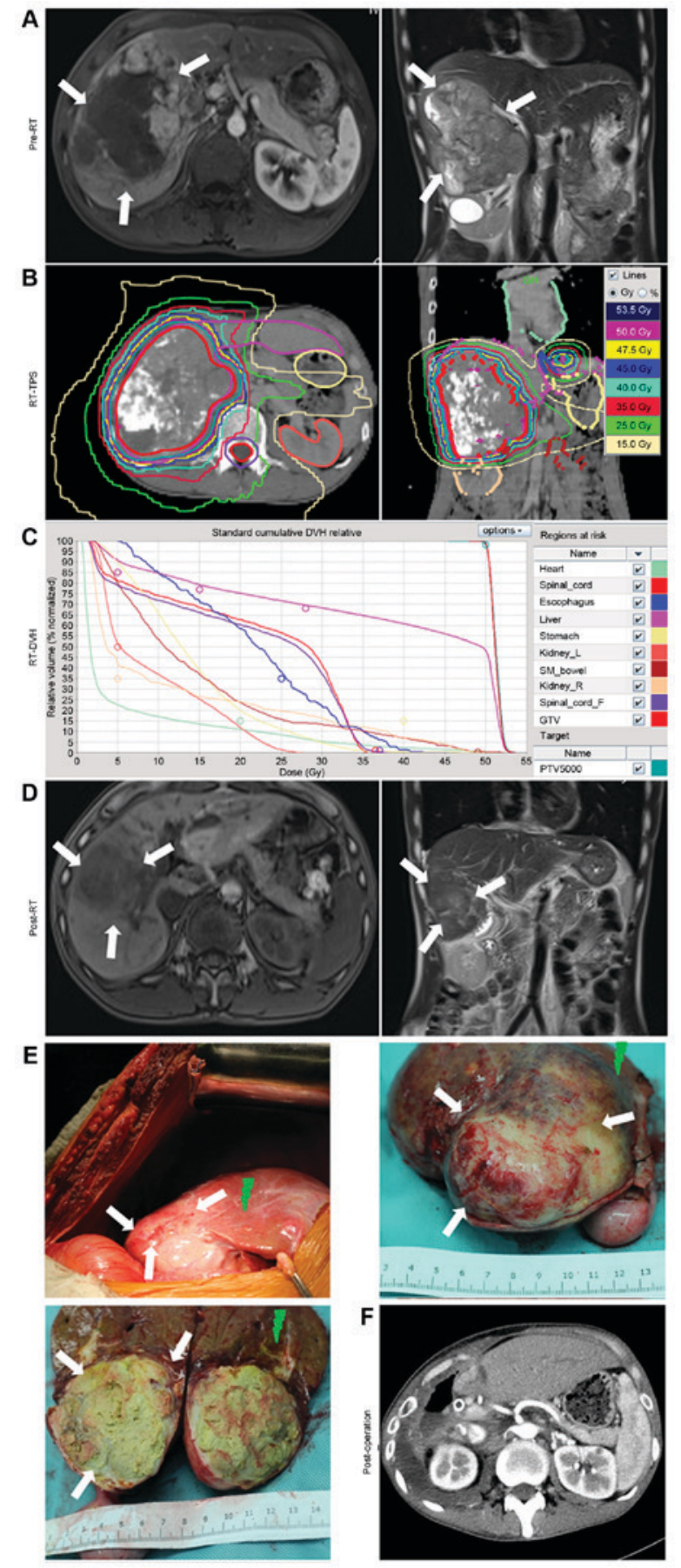

Figure 1. Example of the treatment of a patient with locally advanced hepatocellular carcinoma using RT followed by resective surgery. (A) Pre-RT MRI revealed a large mass signal on the enhanced T1-W1. (B) Isodose distribution graphs for RT, as produced by the RT-TPS computers. The tumor, liver, stomach, esophagus, heart, kidneys and spinal cord were delineated. (C) RT-DVH graph of the tumor, liver, stomach, esophagus, heart, kidneys and spinal cord, as calculated using the TPS. (D) Pre-operative MRIs revealed that the tumor had regressed post-RT, and was now suited for further treatment with surgery. (E) During surgery, it was identified that the remaining tumor had been almost eliminated by RT, with a distinct boundary (white arrows). Radiation-induced liver diseases occurred in the swelling and bleeding zone of the peritumoral liver tissues (green arrows). (F) Computed tomography scans revealed the absence of the right liver lobe and tumor tissue following surgery. RT, radiotherapy; MRI, magnetic resonance imaging; TPS, treatment planning system; DVH, dose volume histogram.

RT. The RT dose to the tumor was $53.5 \mathrm{~Gy}$ in 25 fractions (Fig. 1B and C). At 2 months after RT, the intrahepatic tumor 
had markedly regressed and was judged to be suitable for surgical treatment (Fig. 1D). The surgical resection of the intrahepatic tumors was performed at Zhongshan Hospital (Fig. 1E). A postoperative CT scan was then performed (Fig. 1F).

Tissue microarray (TMA). The TMAs were constructed as previously described (9). Briefly, slides with hematoxylin and eosin (H\&E)-stained sections were screened for optimal tumor and peritumoral liver tissues for the construction of TMAs. Two tissue cores of $2.0 \mathrm{~mm}$ diameter were punched from the non-necrotic areas of the peritumoral liver tissue adjacent to the tumor and tumor foci in formalin-fixed, paraffin-embedded samples. Slides containing $4-\mu \mathrm{m}$ sections were constructed from the resulting TMA blocks. In order to identify the irradiation dose of each tissue section selected for the TMA, the process was directed by the RT isodose distribution graphs from the TPS computers.

Histological evaluation (IHC and $H \& E$ ). TMA sections were stained with $\mathrm{H} \& \mathrm{E}$, or subjected to IHC with antibodies against TLR4 (dilution, 1:100), TRAIL (dilution, 1:50) or VEGFR2 (dilution, 1:50; Abcam; Cambridge, UK), and were visualized with GT Vision III Detection System/Mo and Rb kit (Gene Tech Biotechnology Co., Ltd., Shanghai, China), according to the manufacturer's instructions. H\&E-stained or IHC-stained TMA sections (magnification, $\mathrm{x} 100$ or $\mathrm{x} 400$ ) were evaluated by three of the authors, who were blinded to the patient outcomes. H\&E-stained TMA sections were used to compare the severity of RILDs in patients, scored with the Fudan University Acute RILD Histological system (9). Five high-power fields were randomly selected to evaluate the expression levels of TLR4, TRAIL and VEGFR2.

Based on the staining percentage and intensity of positive cells counted in each core $(9,15)$, the final IHC staining scores were defined as $(-),(+),(++)$ and $(+++)$, which represented a negative, weak, moderate and strong immunoreactivity, respectively. For statistical analysis, the staining scores of cells expressing TLR4, TRAIL and VEGFR-2 were categorized as follows: For TLR4, (-) or (+), low expression; (++) or (+++), high expression; for TRAIL and VEGFR-2, (-), low expression; (+), $(++)$ or $(+++)$, high expression. The patients were divided into high- and low-TLR4, TRAIL and VEGFR-2 expression groups based on the tumor and peritumoral liver tissue expression.

Follow-up. All patients were followed up at the outpatient clinic or via telephone interviews following surgery. Follow-up continued until October 2014. No patient mortality was attributed to surgical complications. All patients underwent the surgical resection of HCC, which was defined as the complete macroscopic removal of the tumor. Clinical information was collected from the computerized data base of Zhongshan Hospital. The overall survival (OS) time was defined as the time from the initial diagnosis date of HCC to the date of mortality or the last follow-up. The disease-free survival (DFS) time was defined as the interval between the date of surgery and the first diagnosis of postoperative HCC recurrence or metastasis, or the last follow-up.

Statistical analysis. Statistical analyses were performed with SPSS 17.0 software (SPSS, Inc., Chicago, IL, USA). OS and
DFS are presented as the median \pm standard error of the mean (SEM); other values are presented as the means \pm SEM. DFS and OS were analyzed using the Kaplan-Meier method. Differences in survival were assessed with the log-rank test. Differences in quantitative data, including the RILD score, were evaluated by t-tests. Differences in frequency data, including the high TLR4 expression ratio of patients, were evaluated by Fisher's exact test. $\mathrm{P}<0.05$ was considered to indicate a statistically significant difference.

\section{Results}

Clinical features and potential biomarker expression in TMA. Following resective surgery, there was typically a distinct tumor boundary (white arrows, Fig. 1E). RILDs typically occurred in the swelling and bleeding zone of peritumoral liver tissues (green arrows, Fig. 1E).

Clinical features, including the TACE treatment history and the DFS and OS time of each patient, are included in Table I. A total of $18(90 \%)$ of the 20 patients were treated with TACE pre- or post-RT. In addition, $4(27 \%)$ of the 15 patients who experienced postoperative disease progression were treated with TACE. The median OS time was $39 \pm 3.17$ months (range, 13-91 months) for the 20 patients. The OS rates at 24 and 36 months were 83.1 and $58.8 \%$, respectively.

According to the results of IHC, TLR4 expression was primarily observed on the membrane and in the cytoplasm of tumor cells and hepatocytes, whereas VEGFR-2 and TRAIL expression were identified in the cytoplasm and on the nuclear membrane (black arrows; all areas of positive staining, Figs. 2 and 3). Of the 20 specimens, 9 (45\%), $8(40 \%)$ and $13(65 \%)$ of the 20 patients exhibited high TLR4, VEGFR2 and TRAIL expression in tumor tissue, respectively; whereas high TLR4, VEGFR2 and TRAIL expression of the peritumoral liver tissue was identified in $9(45 \%), 9(45 \%)$ and $10(50 \%)$ of the specimens, respectively.

Association of OS and DFS with TLR4, VEGFR2 and TRAIL expression in tumor tissue. The OS and DFS times of the patients from the present study are listed in Table I. Kaplan-Meier survival curves are included in Fig. 2. No significant differences were identified between the OS times of patients with high and low tumor tissue VEGFR2 expression $(\mathrm{P}=0.114)$. However, patients with low TLR4 or TRAIL expression in tumor tissue had significantly improved overall survival outcomes vs. those with high TLR4 $(\mathrm{P}=0.003)$ or TRAIL ( $\mathrm{P}=0.007)$ expression (Table II).

There was no significant difference in DFS time between the patients with high and low tumor tissue TLR4 expression $(\mathrm{P}=0.128)$, whereas the DFS of patients with low tumor VEGFR2 or TRAIL expression was significantly longer than those with high tumor VEGFR2 $(\mathrm{P}=0.003)$ or TRAIL $(\mathrm{P}=0.008)$ expression (Fig. 2; Table II).

In summary, longer OS times were observed in patients with tumors with low TLR4 or TRAIL expression; whereas longer DFS times were observed in those with tumors with low VEGFR2 or TRAIL expression (Fig. 2; Table II). These results demonstrate that the level of TLR4 and TRAIL expression in HCC tumor tissues may have a prognostic value for OS, whereas the VEGFR2 or TRAIL expression of tumor tissues 


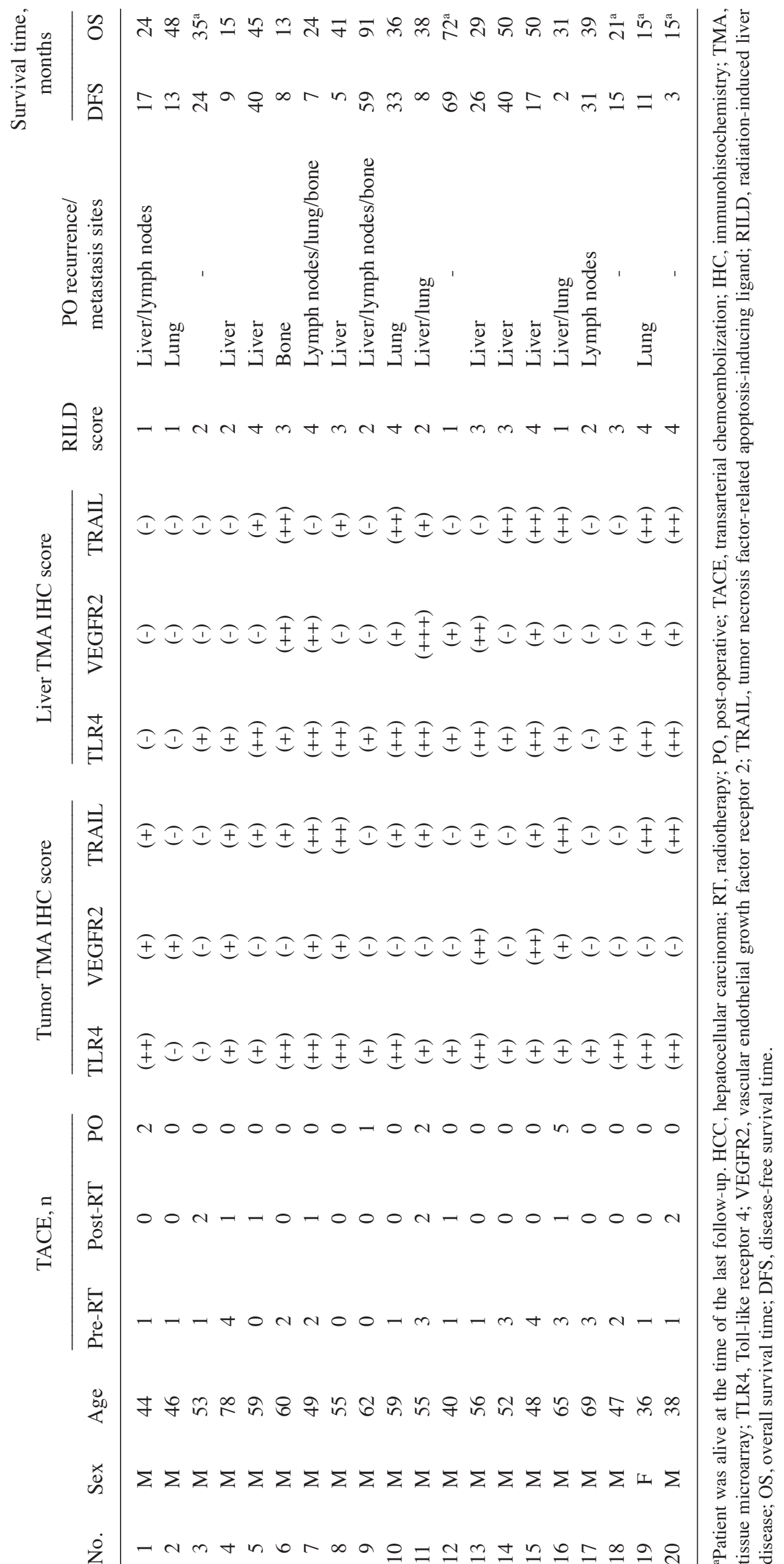



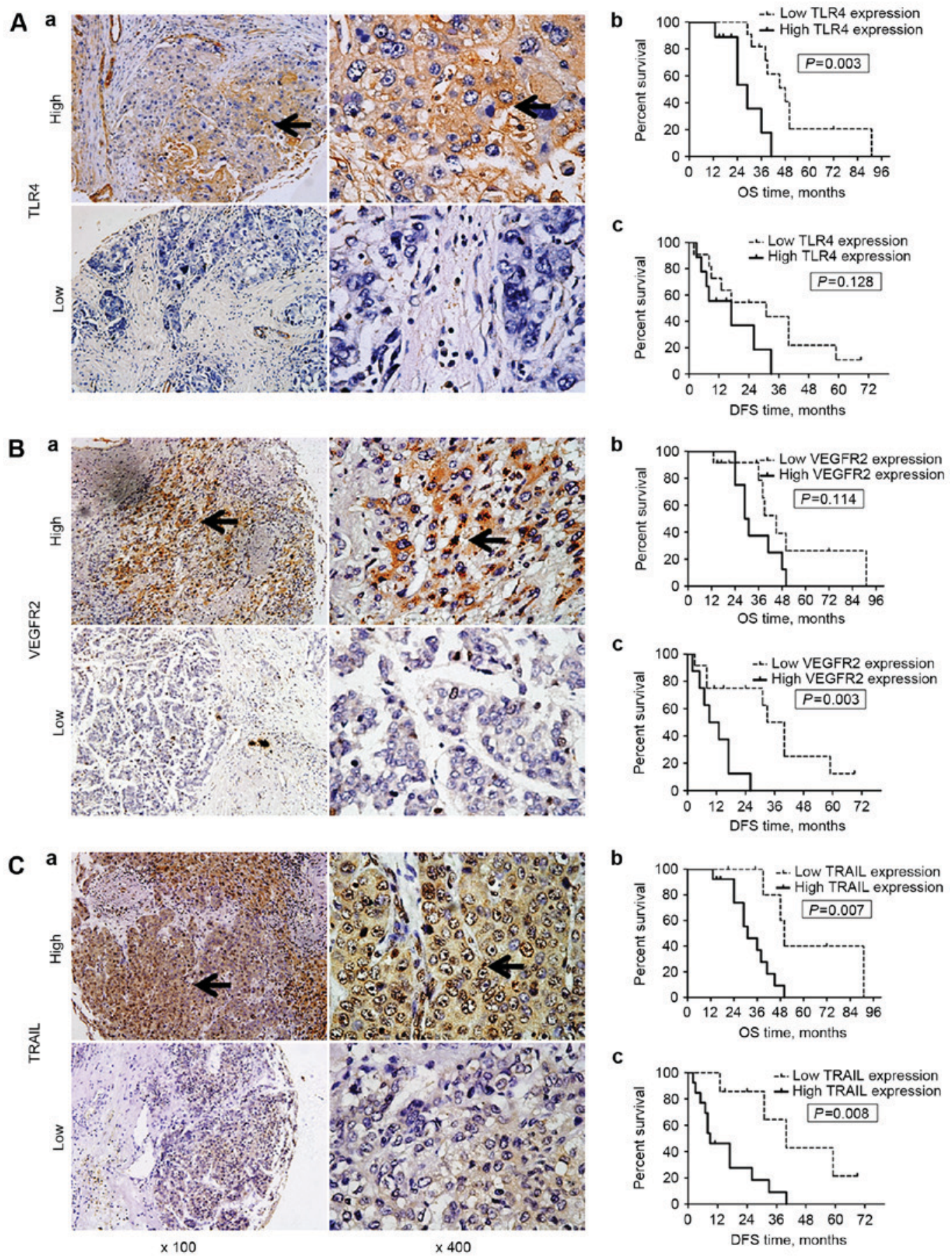

Figure 2. Expression of TLR4, VEGFR2 and TRAIL expression in hepatocellular carcinoma tissue samples, and associated OS/DFS curves. TLR4 expression was primarily observed on the membrane and in the cytoplasm of cells, whereas VEGFR-2 and TRAIL expression were identified in the cytoplasm and on the nuclear membrane (black arrows; all areas of positive IHC staining). (A) TLR4 and OS/DFS. (a) Representative images of high ( $n=9)$ and low ( $n=11)$ TLR4 expression in tumors. (b) OS and (c) DFS curves for the patients stratified by the tumor TLR4 expression level. Patients with low tumor TLR4 expression exhibited a significantly longer OS ( $\mathrm{P}=0.003)$, but not DFS ( $\mathrm{P}=0.128)$ time than those with high TLR4 expression. (B) VEGFR2 and OS/DFS. (a) Representative images of high $(n=8)$ and low $(n=12)$ VEGFR2 expression in tumors. (b) OS and (c) DFS curves for the patients stratified by the tumor VEGFR2 expression level. Patients with low tumor VEGFR2 expression exhibited a significantly longer DFS ( $\mathrm{P}=0.003)$, but not OS ( $\mathrm{P}=0.114)$ time than those with high VEGFR2 expression. (C) TRAIL and OS/DFS. (a) Representative images of high ( $\mathrm{n}=13$ ) and low TRAIL ( $\mathrm{n}=7$ ) expression in tumors. (b) OS and (c) DFS curves for the patients stratified by the tumor TRAIL expression level. Patients with low tumor TRAIL expression exhibited significantly longer DFS ( $\mathrm{P}=0.008)$ and OS (P=0.007) times than those with high TRAIL expression. Magnification, $\mathrm{x} 100$ or $\mathrm{x} 400$. TLR4, Toll-like receptor 4; VEGFR2, vascular endothelial growth factor receptor 2; TRAIL, tumor necrosis factor-related apoptosis-inducing ligand; OS, overall survival; DFS, disease-free survival; IHC, immunohistochemistry.

were associated with the DFS of the patients with HCC treated by surgical resection post-RT.

TLR4, VEGFR2 and TRAIL expression in the peritumoral liver tissues are associated with RILDs, but not with survival.
As demonstrated in Fig. 4 and Table II, the median OS and DFS times for the patients with low TLR4, VEGFR2 or TRAIL expression in peritumoral liver tissue were not significantly different from the times for the patients with high peritumoral TLR4, VEGFR2 or TRAIL expression. 


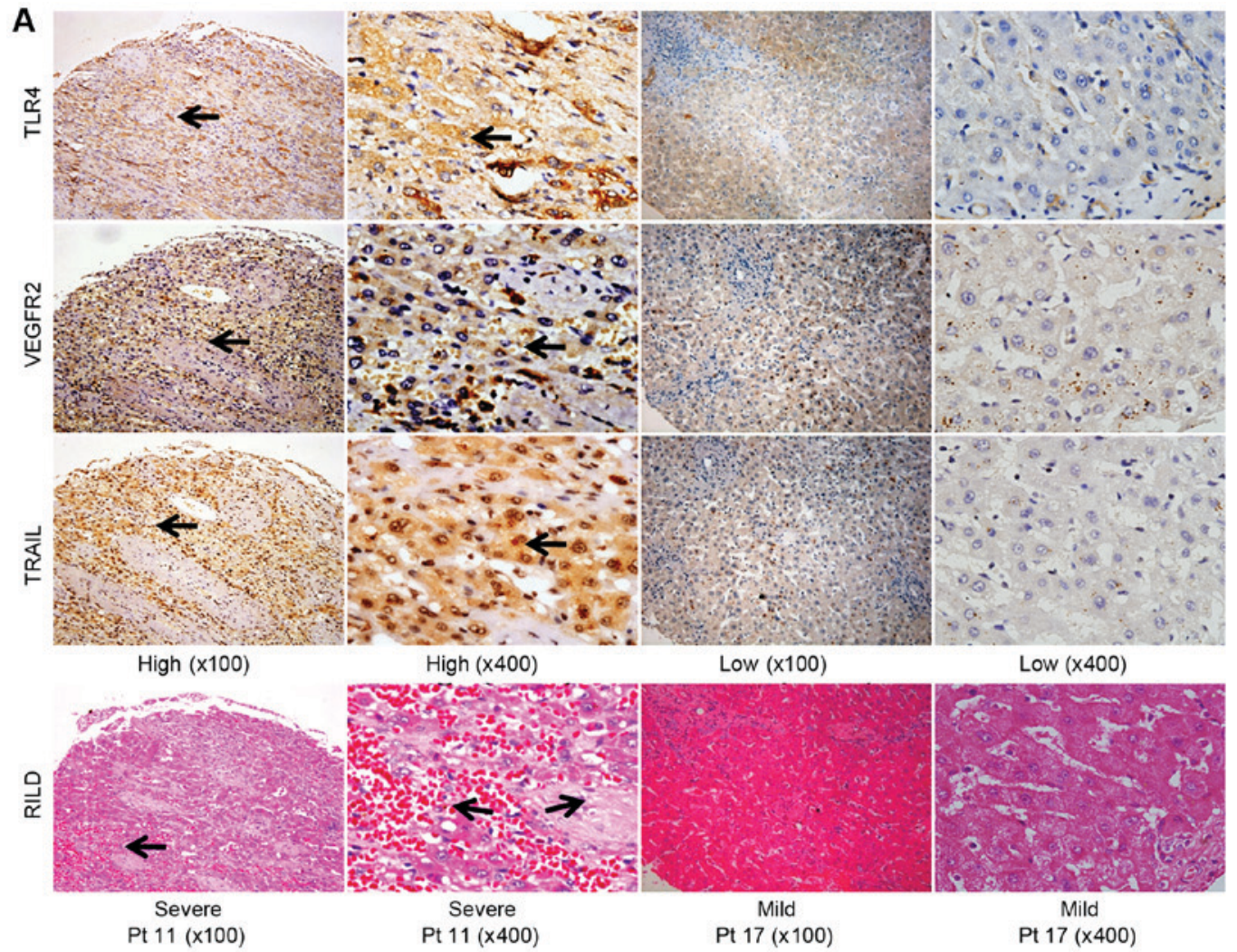

B

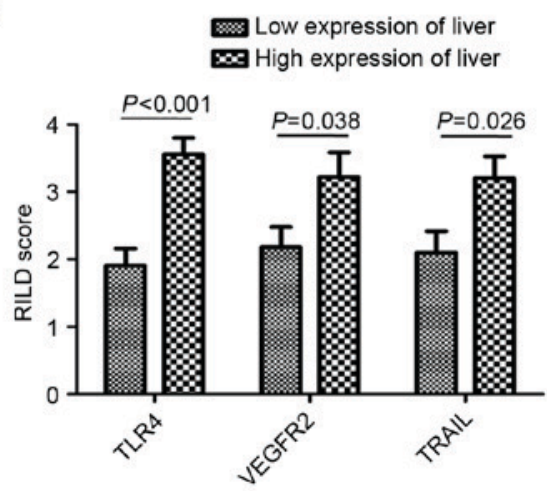

C

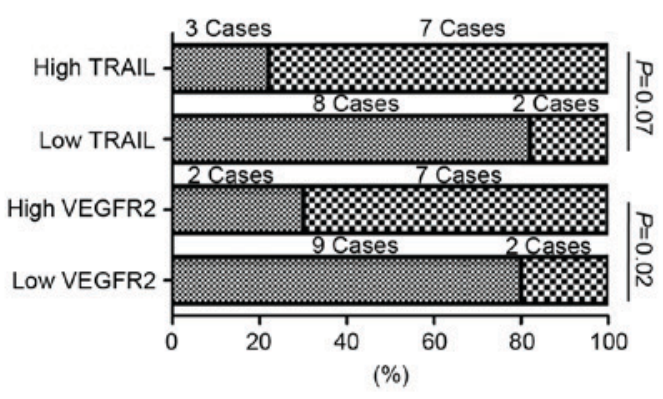

Figure 3. Association of the non-tumor liver tissue expression of TLR4, VEGFR2 and TRAIL with RILDs in patients with HCC treated with RT. (A) Representative images of IHC and H\&E-stained tissue microarrays from 20 patients with HCC. High TLR4, VEGFR2 or TRAIL expression in liver tissue following RT was associated with more severe RILDs. TLR4 expression was primarily observed on the membrane and in the cytoplasm of cells, whereas VEGFR-2 and TRAIL expression were identified in the cytoplasm and on the nuclear membrane (black arrows; all areas of positive IHC-staining). Magnification, x100 or x400. (B) Comparing RILD scores by TLR4, VEGFR2 and TRAIL expressions levels. The mean RILD scores of patients with high TLR4, VEGFR2 or TRAIL expression were significantly higher than the patients with the corresponding low expression level in non-tumor liver tissue $(\mathrm{P}<0.05)$. (C) Association between TLR4 and VEGFR2 or TRAIL expression in the liver post-RT. The rate of high TLR4 expression tended to be higher in patients with high TRAIL expression in the non-tumor liver tissue, whereas significantly more patients with high TLR4 expression were identified in the group with high VEGFR2 expression in the non-tumor liver tissue post-RT. TLR4, Toll-like receptor 4; VEGFR2, vascular endothelial growth factor receptor 2; TRAIL, tumor necrosis factor-related apoptosis-inducing ligand; RILD, radiation-induced liver disease; HCC, hepatocellular carcinoma; RT, radiotherapy; IHC, immunohistochemistry; H\&E, hematoxylin and eosin.

These results demonstrate that the levels of TLR4, VEGFR2 or TRAIL expression in peritumoral liver tissues may not be suitable as biomarkers for survival outcomes for patients with HCC receiving surgical resection following RT.

RILDs resulting from the irradiation of involved normal liver tissue were assessed and scored in the H\&E-stained liver TMA slides. Patients with higher TLR4 expression developed more severe RILDs (RILD score, $3.56 \pm 0.24$ vs. $1.91 \pm 0.25$; $\mathrm{P}<0.001$ ), which indicated that severe RILDs may have been induced by the TLR4-dependent response to the RT treatment of HCC. This was also observed in patients with high peritumoral TRAIL or VEGFR2 expression (RILD scores, $3.20 \pm 0.33$ and $3.22 \pm 0.36$, respectively) compared to those with low expression (RILD scores, $2.10 \pm 0.31$ and 2.18 \pm 0.30 , respectively; $\mathrm{P}<0.05$; Fig. $3 \mathrm{~A}$ and $\mathrm{B}$ ).

There were 2 patients with high TLR4 expression that also presented with low VEGFR2 expression (2/11), whereas 7 patients with high VEGFR2 expression also exhibited high TLR4 expression (7/9) in the peritumoral liver tissues post-RT $(\mathrm{P}=0.02$; Fig. 3C). Although no significant differences were 
Table II. Correlations between TLR4/VEGFR2/TRAIL expression and survival results of HCC patients undergoing hepatectomy post-RT.

\begin{tabular}{|c|c|c|c|c|c|c|c|c|}
\hline \multirow[b]{2}{*}{ Expression } & \multicolumn{4}{|c|}{ Median OS time } & \multicolumn{4}{|c|}{ Median DFS time } \\
\hline & Low, months & High, months & $\chi^{2}$ & P-value & Low, months & High, months & $\chi^{2}$ & P-value \\
\hline \multicolumn{9}{|l|}{ TLR4 } \\
\hline Tumor & $48 \pm 6.86(n=11)$ & $29 \pm 5.98(n=9)$ & 8.854 & 0.003 & $31 \pm 13.93$ & $17 \pm 9.10$ & 2.321 & 0.128 \\
\hline Liver & $39 \pm 11.51(\mathrm{n}=11)$ & $38 \pm 2.62(\mathrm{n}=9)$ & 0.872 & 0.351 & $31 \pm 12.32$ & $17 \pm 11.21$ & 1.648 & 0.199 \\
\hline \multicolumn{9}{|l|}{ VEGFR2 } \\
\hline Tumor & $45 \pm 4.67(n=12)$ & $29 \pm 3.30(n=8)$ & 2.497 & 0.114 & $33 \pm 4.00$ & $9 \pm 4.24$ & 8.585 & 0.003 \\
\hline Liver & $41 \pm 4.26(n=11)$ & $36 \pm 8.92(\mathrm{n}=9)$ & 0.189 & 0.664 & $31 \pm 12.32$ & $17 \pm 11.21$ & 0.114 & 0.736 \\
\hline \multicolumn{9}{|l|}{ TRAIL } \\
\hline Tumor & $50 \pm 2.91(\mathrm{n}=7)$ & $31 \pm 5.71(n=13)$ & 7.348 & 0.007 & $40 \pm 9.42$ & $9 \pm 3.33$ & 7.04 & 0.008 \\
\hline Liver & $39 \pm 12.45(\mathrm{n}=10)$ & $41 \pm 3.37(n=10)$ & 0.26 & 0.61 & $26 \pm 10.78$ & $8 \pm 5.83$ & 1.526 & 0.217 \\
\hline
\end{tabular}

HCC, hepatocellular carcinoma; RT, radiotherapy; TLR4, Toll-like receptor 4; VEGFR2, vascular endothelial growth factor receptor 2; TRAIL, tumor necrosis factor-related apoptosis-inducing ligand; OS, overall survival time; DFS, disease-free survival time.

identified between the patients with low and high TRAIL expression $(\mathrm{P}=0.07)$, the number of patients with high TLR4 expression was reduced in the group of patients with low TRAIL expression in the peritumoral liver tissue post-RT (Fig. 3C). These findings demonstrated that high TLR4 expression maybe associated with severe RILDs and high VEGFR2 or TRAIL expression in post-RT peritumoral liver tissues.

\section{Discussion}

Novel therapeutic strategies continue to be trialed at Zhongshan Hospital, with the aim of improving the outcome for patients with inoperable and/or locally advanced HCC. Assuring clinical target volume coverage and performing advanced RT delivery techniques, including intensity modulated RT and helical tomotherapy, can minimize toxicity to the normal liver tissue while continuing to deliver an effective treatment for HCC $(16,17)$. In a previous study at Zhongshan Hospital, OS rates for patients with HCC treated by RT were 42.3 and $24.0 \%$ at 2 and 3 years, respectively, whereas patients that did not receive RT exhibited OS rates of 26.5 and $11.1 \%$ at 2 and 3 years, respectively (18). Furthermore, certain patients with inoperable HCC gained a secondary opportunity for surgical resection when the tumor size had reduced subsequent to RT. Improved survival outcomes were also identified in patients with HCC treated by surgical resection post-RT (OS rates, 83.1 and $58.8 \%$ at 2 and 3 years, respectively) compared with those treated by RT without surgery, which may be due to the RT-mediated effect of the elimination of cancer cells that had spread into the liver tissue around tumors. Although survival benefits were demonstrated in our previous studies, not every patient with inoperable HCC was benefited by post-RT surgical resection. The outcome for certain patients remains unsatisfactory; for example, in the present study, the lowest OS and DFS times for the patients were 13 and 2 months, respectively. Therefore, it is important to identify predictors to estimate the prognosis of patients with $\mathrm{HCC}$ that are treated with surgery post-RT.
The TLR4-dependent immune response is a critical constituent of innate immunity $(19,20)$. TLR4 maybe expressed not only in healthy liver cells, including hepatocytes and hepatic stellate cells (6), but also in certain types of tumor cells, including in HCC. For example, it has been observed that TLR4 may be stimulated by DAMPs to promote the growth of HCC (21). Yu et al (22) hypothesized that blocking the TLR4-mediated signaling pathway may inhibit HCC invasion and metastasis. By examining the TLR4 expression level in tumors from 30 patients with HCC, Eiró et al (23) identified that positive TLR4 immunostaining was associated with a relatively poor prognosis. In the present study, it was identified that the OS time of patients with low tumor TLR4 expression was significantly longer than those with high tumor TLR4 expression. Therefore, the upregulation of TLR4 in HCC tissue may contribute to the poor prognosis of certain patients with HCC treated with post-RT surgery.

A number of studies have identified that distinct cytokine responses are associated with radiation-induced inflammation $(24,25)$. The inhibition of TRAIL has been demonstrated to specifically eliminate malignant cells, which can be combined with RT to further enhance the anticancer effect (26). Similarly, a significantly improved OS and DFS time was observed in patients with low tumor TRAIL expression compared with patients with high tumor TRAIL expression in the present study.

VEGFR2 is one of the most critical receptors for VEGF (27); tumor angiogenesis can be inhibited by blocking the activity of VEGFR2 $(27,28)$. Due to escalating the rate of vascular repair, radiation-induced upregulation of VEGFR2 in cancer cells may contribute to RT failure (29). In the present study, it was identified that the DFS time was significantly longer in patients with low VEGFR2 expression in HCC tumor tissue following post-RT surgery. Therefore, the expression levels of TRAIL or VEGFR2 in HCC tissue may be suitable for use as predictive factors for patients with HCC treated by post-RT surgery.

The excessive activation of TLR4 signaling may induce liver damage (8-10). Although the expression levels of TLR4, 
A

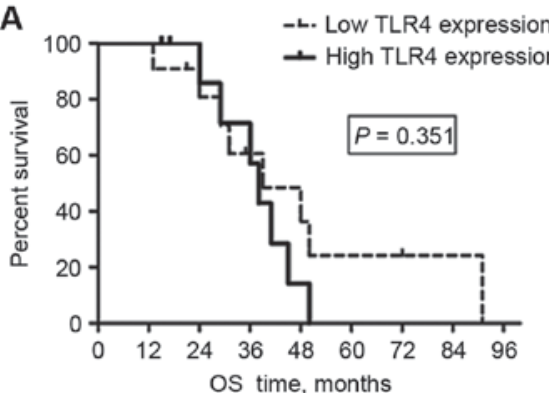

C

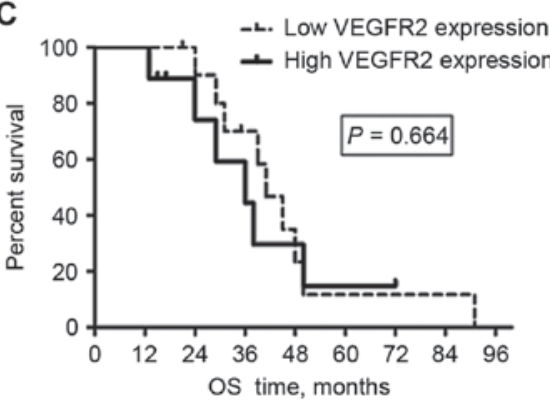

E

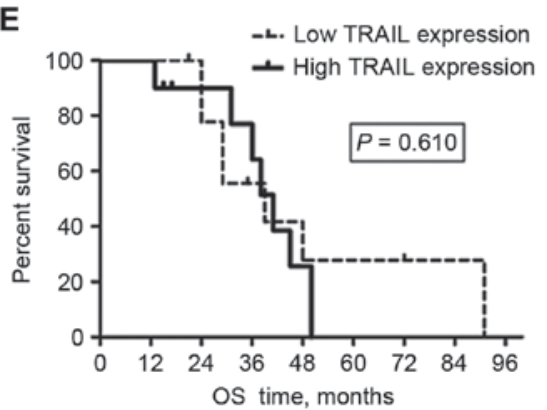

B

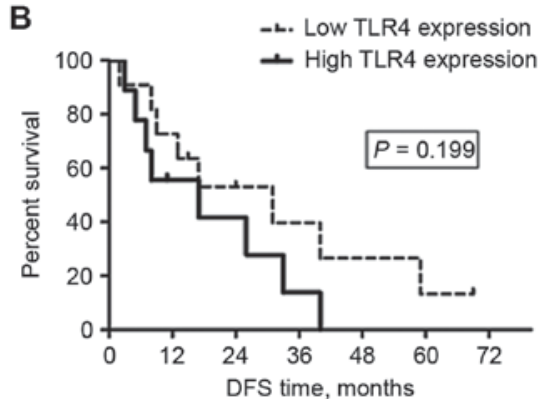

D

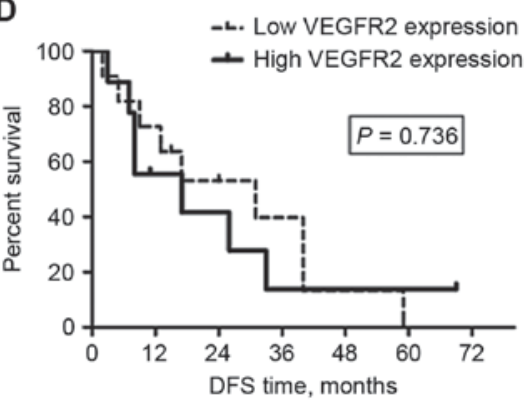

$\mathbf{F}$

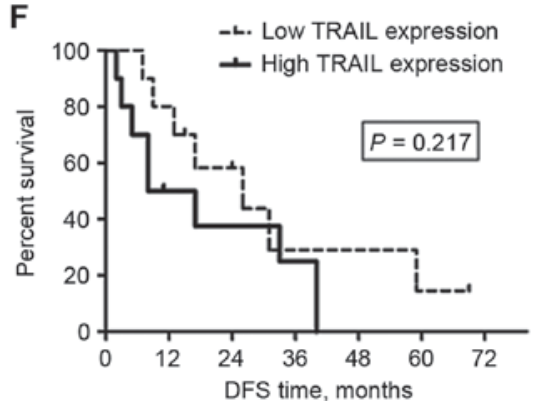

Figure 4. OS and DFS times for patients with hepatocellular carcinoma treated by post-radiotherapy surgery were not associated with the TLR4, VEGFR2 and TRAIL expression levels of the non-tumor liver tissue. (A) OS and (B) DFS were not associated with the TLR4 expression of the liver. (C) OS and (D) DFS were not associated with the VEGFR2 expression of the liver. (E) OS and (F) DFS were not associated with the TRAIL expression of the liver. OS, overall survival; DFS, disease-free survival; TLR4, Toll-like receptor 4; VEGFR2, vascular endothelial growth factor receptor 2; TRAIL, tumor necrosis factor-related apoptosis-inducing ligand.

VEGFR2 and TRAIL in liver tissue were not identified as predictors for survival outcomes in patients with HCC treated by post-RT surgery, the results of the present study indicated that they were associated with the severity of RILDs, potentially fatal complications that restrict radiotherapeutic efficacy against HCC, including liver piecemeal necrosis, inflammation, hepatic veno-occlusive disease and sinusoidal obstruction syndrome $(30,31)$. In the present study, patients with low TLR4 expression presented with milder RILDs than those with high TLR4 expression in the peritumoral liver tissue subsequent to liver RT, which was consistent with the preliminary data of another study of ours, in mice (10). TRAIL not only promotes the malignant behavior of cancer cells, but is also associated with hepatocyte toxicity when combined with RT $(26,32)$. VEGFR2 is widely distributed throughout human tissue, including tumors (33). According to the IHC and H\&E histological findings from the present study, higher VEGFR-2 and TRAIL expression tended to be associated with high TLR4 expression in liver tissue. More severe RILDs in the surrounding liver tissue were also identified in patients with high VEGFR-2 and TRAIL expression in the liver compared with those with low expression.
In addition, $90 \%$ of the patients in the present study were treated with TACE pre- or post-RT. TACE is typically used for the palliation of unresectable HCC, with a significant tumor response rate of $17-61.9 \%$, although the complete tumor response rate to TACE is unsatisfactory $(0-4.8 \%)$ (13). TACE can also serve as abridging therapy prior to RT. In order to avoid RILD, the minimum normal liver volume (minus the gross tumor volume) is defined as $700 \mathrm{ml}$. HCC patients with particularly large tumors can only be treated with RT when significant tumor regression has occurred, and normal liver volume is sufficient, post-TACE $(34,35)$. A previous study has also demonstrated that TACE with RT, compared with TACE alone or RT alone, improved the survival outcomes of patients with unresectable HCC (36). Shim et al (37) reported that the 2-year survival rate of HCC patients treated by TACE and RT was significantly higher than patients treated by TACE alone (36.8 vs. $14.3 \%, \mathrm{P}=0.001$ ), particularly in cases of tumors $\geq 8 \mathrm{~cm}$ diameter. Both of these rates are lower than the 2-year survival rate of patients treated by surgical resection post-RT in the present study (83.1\%), despite $18 / 20$ of the patients receiving TACE treatment, which indicates that 
TACE may not have affected the survival outcome for patients in the present study. Statistical analysis of the data from the present study demonstrated that there were no differences in the TACE treatment status between patients with high and low TLR4, VEGFR2 or TRAIL expression in liver or tumor tissues in the present study, respectively (all P>0.05). This further illustrated that pre- or post-RT TACE treatment did not affect the survival outcomes of the patients in the present study.

In summary, patients with stage III HCC, particularly patients with low tumor TLR4, VEGFR2 or TRAIL expression, may benefit from treatment with surgical resection post-RT. In addition, the high expression of TLR4, VEGFR2 or TRAIL in the peritumoral liver tissue was associated with more severe RILDs, but not with the prognosis of patients with HCC treated by post-RT surgery. Therefore, therapeutic approaches of inhibiting TLR4, VEGFR2 or TRAIL in liver and HCC tissue may prevent or lessen RILDs, and improve the survival outcomes of patients with HCC treated by post-RT surgery in the future.

\section{Acknowledgements}

Not applicable.

\section{Funding}

The present study was supported by the National Natural Science Foundation of China (grant no. U1505229), the Doctoral Foundation of Education Department of China (grant no. 20120071110065) and the Science and Technology Innovation Plan of Shanghai (grant no. 14140902303).

\section{Availability of data and materials}

The data and materials of the current study are available from the corresponding author on reasonable request.

\section{Authors' contributions}

ZFW performed the histological examination of the tissue microarrays. The overall and disease-free survival outcomes for each patient were assessed by YW. PY applied the resected HCC tissues and peritumoral liver tissues to construct TMAs. They were major contributors in writing the manuscript. The severity of RILDs was detected by JZH. JYZ analyzed and interpreted the patient data regarding the intrahepatic tumors treated with EBRT. All patients were followed up by YH. ZCZ made contributions to conception and design of the research, and gave final approval of the version to be published. All authors have read and approved the final manuscript.

\section{Ethics approval and consent to participate}

The study followed international and national regulations in accordance with the Declaration of Helsinki, and was approved by the Zhongshan Hospital Research Ethics Committee. All patients provided written consent for their inclusion in research.

\section{Consent for publication}

Identifying information of patients in our manuscript is essential for scientific purposes and will be treated confidentially. Patients or guardians have provided written informed consent for publication of any associated data and accompanying images.

\section{Competing interests}

The authors declare that they have no competing interests.

\section{References}

1. Stroescu C, Dragnea A, Ivanov B, Pechianu C, Herlea V, Sgarbura O, Popescu A and Popescu I: Expression of p53, Bcl-2, VEGF, Ki67 and PCNA and prognostic significance in hepatocellular carcinoma. J Gastrointestin Liver Dis 17: 411-417, 2008.

2. Price TR, Perkins SM, Sandrasegaran K, Henderson MA Maluccio MA, Zook JE, Tector AJ, Vianna RM, Johnstone PA and Cardenes HR: Evaluation of response after stereotactic body radiotherapy for hepatocellular carcinoma. Cancer 118: 3191-3198, 2012.

3. Law AL, Ng WT, Lee MC, Chan AT, Fung KH, Li F, Lao WC and Lee AW: Treatment of primary liver cancer using highlyconformal radiotherapy with $\mathrm{kV}$-image guidance and respiratory control. Radiother Oncol 102: 56-61, 2012.

4. Chen D, Wang R, Meng X, Yan H, Jiang S, Feng R, Zhu K, Xu X, Dou $X$ and Jin L: Prognostic value of serum $\gamma$-glutamyl transferase in unresectable hepatocellular carcinoma patients treated with transcatheter arterial chemoembolization combined with conformal radiotherapy. Oncol Lett 8: 2298-2304, 2014.

5. Apetoh L, Ghiringhelli F, Tesniere A, Obeid M, Ortiz C, Criollo A, Mignot G, Maiuri MC, Ullrich E, Saulnier P, et al: Toll-like receptor 4-dependent contribution of the immune system to anticancer chemotherapy and radiotherapy. Nat Med 13: 1050-1059, 2007.

6. Seki E and Brenner DA: Toll-like receptors and adaptor molecules in liver disease: Update. Hepatology 48: 322-335, 2008.

7. Hiratsuka S, Watanabe A, Sakurai Y, Akashi-Takamura S, Ishibashi S, Miyake K, Shibuya M, Akira S, Aburatani $\mathrm{H}$ and Maru Y: The S100A8-serum amyloid A3-TLR4 paracrine cascade establishes a pre-metastatic phase. Nat Cell Biol 10: 1349-1355, 2008.

8. Huang B, Zhao J, Li H, He KL, Chen Y, Chen SH, Mayer L, Unkeless JC and Xiong H: Toll-like receptors on tumor cells facilitate evasion of immune surveillance. Cancer Res 65: 5009-5014, 2005.

9. Wu ZF, Zhou XH, Hu YW, Zhou LY, Gao YB, Peng XH, Yang XH, Zhang JY, Hu Y and Zeng ZC: TLR4-dependant immune response, but not hepatitis B virus reactivation, is important in radiation-induced liver disease of liver cancer radiotherapy. Cancer Immunol Immunother 63: 235-245, 2014.

10. Zhi-Feng W, Le-Yuan Z, Xiao-Hui Z, Ya-Bo G, Jian-Ying Z, Yong $\mathrm{H}$ and Zhao-Chong $\mathrm{Z}$ : TLR4-dependent immune response promotes radiation-induced liver disease by changing the liver tissue interstitial microenvironment during liver cancer radiotherapy. Radiat Res 182: 674-682, 2014.

11. Ramacciato G, Mercantini P, Cautero N, Corigliano N, Di Benedetto F, Quintini C, Ercolani G, Varotti G, Ziparo V and Pinna AD: Prognostic evaluation of the new American Joint Committee on Cancer/International Union Against Cancer staging system for hepatocellular carcinoma: Analysis of 112 cirrhotic patients resected for hepatocellular carcinoma. Ann Surg Oncol 12: 289-297, 2005.

12. Hodi FS, Ballinger M, Lyons B, Soria JC, Nishino M, Tabernero J, Powles T, Smith D, Hoos A, McKenna C, et al: Immune-modified response evaluation criteria in solid tumors (imRECIST): Refining guidelines to assess the clinical benefit of cancer immunotherapy. J Clin Oncol 36: 850-858, 2018.

13. Ma S, Jiao B, Liu X, Yi H, Kong D, Gao L, Zhao G, Yang Y and Liu X: Approach to radiation therapy in hepatocellular carcinoma. Cancer Treat Rev 36: 157-163, 2010.

14. Galle PR, Tovoli F, Foerster F, Wörns MA, Cucchetti A and Bolondi L: The treatment of intermediate stage tumours beyond TACE: From surgery to systemic therapy. J Hepatol 67: 173-183, 2017. 
15. Xu H, Wu Q, Dang S, Jin M, Xu J, Cheng Y, Pan M, Wu Y, Zhang $C$ and Zhang Y: Alteration of CXCR7 expression mediated by TLR4 promotes tumor cell proliferation and migration in human colorectal carcinoma. PLoS One 6: e27399, 2011.

16. Long Z, Wang B, Tao D, Liu Y, Zhang J, Tan J, Luo J, Shi F and Tao Z: Clinical research on alternating hyperfraction radiotherapy for massive hepatocellular carcinoma. Oncol Lett 10: 523-527, 2015

17. vander Schaaf A, Xu CJ, van Luijk P, Van't Veld AA, Langendijk JA and Schilstra C: Multivariate modeling of complications with data driven variable selection: Guarding against overfitting and effects of data set size. Radiother Oncol 105: 115-121, 2012.

18. Zeng ZC, Tang ZY, Fan J, Zhou J, Qin LX, Ye SL, Sun HC, Wang BL, Yu Y, Wang JH and Guo W: A comparison of chemoembolization combination with and without radiotherapy for unresectable hepatocellular carcinoma. Cancer J 10: 307-316, 2004

19. Yamamoto M, Sato S, Hemmi H, Hoshino K, Kaisho T, Sanjo H, Takeuchi O, Sugiyama M, Okabe M, Takeda K and Akira S: Role of adaptor TRIF in the MyD88-independent toll-like receptor signaling pathway. Science 301: 640-643, 2003.

20. Schmidt M, Raghavan B, Müller V, Vogl T, Fejer G, Tchaptchet S, Keck S, Kalis C, Nielsen PJ, Galanos C, et al: Crucial role for human Toll-like receptor 4 in the development of contact allergy to nickel. Nat Immunol 11: 814-819, 2010.

21. Wu FH, Yuan Y, Li D, Liao SJ, Yan B, Wei JJ, Zhou YH, Zhu JH, Zhang GM and Feng ZH: Extracellular HSPA1A promotes the growth of hepatocarcinoma by augmenting tumor cell proliferation and apoptosis-resistance. Cancer Lett 317: 157-164, 2012.

22. Yu P, Cheng X, Du Y, Huang L and Dong R: TAK-242 can be the potential agents for preventing invasion and metastasis of hepatocellular carcinoma. Med Hypotheses 81: 653-655, 2013.

23. Eiró N, Altadill A, Juárez LM, Rodríguez M, González LO, Atienza S, Bermúdez S, Fernandez-Garcia B, FresnoForcelledo MF, Rodrigo L and Vizoso FJ: Toll-like receptors 3 , 4 and 9 in hepatocellular carcinoma: Relationship with clinicopathological characteristics and prognosis. Hepatol Res 44: 769-778, 2014.

24. Varnum SM, Springer DL, Chaffee ME, Lien KA, WebbRobertson BJ, Waters KM and Sacksteder CA: The effects of low-dose irradiation on inflammatory response proteins in a 3D reconstituted human skin tissue model. Radiat Res 178: 591-599, 2012.

25. Gallet P, Phulpin B, Merlin JL, Leroux A, Bravetti P, Mecellem H, Tran N and Dolivet G: Long-term alterations of cytokines and growth factors expression in irradiated tissues and relation with histological severity scoring. PLoS One 6: e29399, 2011.

26. Zhang X, Cheung RM, Komaki R, Fang B and Chang JY: Radiotherapy sensitization by tumor-specific TRAIL gene targeting improves survival of mice bearing human non-small cell lung cancer. Clin Cancer Res 11: 6657-6668, 2005
27. Schenone S, Bondavalli $\mathrm{F}$ and Botta $\mathrm{M}$ : Antiangiogenic agents: An update on small molecule VEGFR inhibitors. Curr Med Chem 14: 2495-2516, 2007.

28. Paz K and Zhu Z: Development of angiogenesis inhibitors to vascular endothelial growth factor receptor 2 . Current status and future perspective. Front Biosci 10: 1415-1439, 2005.

29. Solberg TD, Nearman J, Mullins J, Li S and BaranowskaKortylewicz J: Correlation between tumor growth delay and expression of cancer and host VEGF, VEGFR2, and osteopontin in response to radiotherapy. Int J Radiat Oncol Biol Phys 72: 918-926, 2008.

30. Cheng JC, Liu HS, Wu JK, Chung HW and Jan GJ: Inclusion of biological factors in parallel-architecture normal-tissue complication probability model for radiation-induced liver disease. Int J Radiat Oncol Biol Phys 62: 1150-1156, 2005.

31. Huang BS, Tsang NM, Lin SM, Lin DY, Lien JM, Lin CC, Chen WT, Chen WY and Hong JH: High-dose hypofractionated X-ray radiotherapy for hepatocellular carcinoma: Tumor responses and toxicities. Oncol Lett 6: 1514-1520, 2013.

32. Lawrence D, Shahrokh Z, Marsters S, Achilles K, Shih D, Mounho B, Hillan K, Totpal K, DeForge L, Schow P, et al: Differential hepatocyte toxicity of recombinant Apo2L/TRAIL versions. Nat Med 7: 383-385, 2001.

33. Stewart M, Turley H, Cook N, Pezzella F, Pillai G, Ogilvie D, Cartlidge S, Paterson D, Copley C, Kendrew J, et al: The angiogenic receptor KDR is widely distributed in human tissues and tumors and relocates intracellularly on phosphorylation. An immunohistochemical study. Histopathology 43: 33-39, 2003.

34. Hawkins MA and Dawson LA: Radiation therapy for hepatocellular carcinoma: From palliation to cure. Cancer 106: 1653-1663, 2006.

35. Pan CC, Kavanagh BD, Dawson LA, Li XA, Das SK, Miften M and Ten Haken RK: Radiation-associated liver injury. Int J Radiat Oncol Biol Phys 76 (3 Suppl): S94-S100, 2010.

36. Kondo Y, Kimura O and Shimosegawa T: Radiation therapy has been shown to be adaptable for various stages of hepatocellular carcinoma. World J Gastroenterol 21: 94-101, 2015.

37. Shim SJ, Seong J, Han KH, Chon CY, Suh CO and Lee JT: Local radiotherapy as a complement to incomplete transcatheter arterial chemoembolization in locally advanced hepatocellular carcinoma. Liver Int 25: 1189-1196, 2005.

This work is licensed under a Creative Commons Attribution-NonCommercial-NoDerivatives 4.0 International (CC BY-NC-ND 4.0) License. 